\title{
Identifying Nanometer-scale Clustering in InAlAsSb Random Alloys Using Atom Probe Tomography
}

Nicole A. Kotulak ${ }^{1}$, Keith Knipling ${ }^{2}$, Louise C. Hirst ${ }^{2}$, Stephanie Tomasulo², Josh Abell ${ }^{2}$, Maria Gonzalez ${ }^{3}$, Michael K. Yakes ${ }^{2}$, Jerry R. Meyer ${ }^{2}$, Robert J. Walters ${ }^{2}$

1. NRC Postdoctoral Associate residing at the U.S. Naval Research Laboratory, Washington, DC 20375 USA

2. U.S. Naval Research Laboratory, Washington, DC 20375 USA

3. Sotera Defense Solutions, Inc., Herndon, VA 20171 USA

Semiconductor devices with enhanced efficiency, resilience, and/or optimized to specific applications/environments rely increasingly on the incorporation of III-V random alloys that have been engineered to possess certain required optoelectronic properties. When these materials are grown, however, challenges with non-random elemental arrangements can greatly affect - and in some cases eliminate - realization of the desired optoelectronic behavior. Multijunction photovoltaic devices represent a case in point. As efficiencies are pushed to break the $50 \%$ mark, the system of III-V materials lattice-matched to InP, with available direct bandgaps of $0.74-1.8 \mathrm{eV}$, nominally provide a near-ideal bandgap combination for triple-junction (3J) devices [1], [2]. The top junction under consideration for this system is the InAlAsSb random alloy, due to its theoretically predicted maximum bandgap of $1.74 \mathrm{eV}$.

The measured bandgaps of the grown InAlAsSb films are narrower, however, than the predicted values. While secondary ion mass spectrometry (SIMS) and X-ray diffraction confirm that the bulk composition and lattice-matching, respectively, are within measurement error of the target values, photoluminescence measurements indicate a type II band alignment (from a power-dependent blue shift) as well as carrier confinement (from the temperature dependence) [3]. Both systematic reduction of the growth temperature and high-temperature post-growth annealing are found to (re)organize the alloy constituents into a more preferable configuration with increased bandgap [4], [5]. Nonetheless, transmission electron microscopy (TEM) and atom probe tomography (APT) on similar samples indicate the existence of small-magnitude cluster features - likely on a scale tens of angstroms [6], [7]. In the present investigation, we have used APT to identify the size, distribution, and nature of any compositional variations occurring at the sub-nm length scales. The result is a better understanding of the growth kinetics for this and related alloy systems, with the goal of allowing future complex growth challenges to be addressed.

A Varian Mod Gen II molecular beam epitaxy (MBE) system with In, Ga, Al, As, and $\mathrm{Sb}$ sources was used to grow the InAlAsSb alloy samples investigated in this work. First a $200 \mathrm{~nm}$ InGaAs buffer layer was grown at $490^{\circ} \mathrm{C}$, followed by $1 \mu \mathrm{m}$ of InAlAsSb, lattice-matched to the InP substrate, at $325^{\circ} \mathrm{C}$. The composition $\operatorname{In}_{0.31} \mathrm{Al}_{0.69} \mathrm{As}_{0.82} \mathrm{Sb}_{0.18}$ was confirmed by SIMS. While the target theoretical bandgap was $1.57 \mathrm{eV}$ at $300 \mathrm{~K}$, the peak PL emission energy was $1.43 \mathrm{eV}$ [3]. APT specimens were prepared using an FEI Nova 600 NanoLab DualBeam ${ }^{T M}$ FIB/SEM and mounted onto flat-topped Si microtip arrays. A Cameca 4000X Si LEAP was used for APT analysis, with specimen base temperature of $25 \mathrm{~K}, 0.01 \mathrm{pJ}$ nominal laser pulse energy, $125 \mathrm{kHz}$ pulse repetition rate, and a $0.5 \%$ detection rate. Cameca Integrated Visualization and Analysis Software (IVAS) 3.6.14 was used for data reconstruction and analysis. 
A $20 \times 20 \times 65 \mathrm{~nm}^{3}$ region of interest (ROI), where the z-axis is parallel to the growth direction, was selected for quantitative analysis. A frequency distribution analysis, with a bin size of 100 and a significance level of 0.01, indicates that all four main elements - In, Al, As, and Sb - have statistically nonrandom distributions throughout the ROI. The 2D composition contour comparison perpendicular to the growth direction, presented in Fig. 1, indicates that In and As have regions of anti-clustering behavior on the order of $2-8 \mathrm{~nm}$ in size, which is corroborated by a radial distribution analysis. On the other hand, $\mathrm{Al}$ and $\mathrm{Sb}$ do not appear to exhibit any visibly strong clustering or anti-clustering tendencies with respect to the other elements within the view of these $2 \mathrm{D}$ concentration contour maps. We are currently determining the nature of this behavior in analyses parallel to the growth direction, as well as the compositional distribution of the cluster/anti-cluster regions. [8]

\section{References:}

[1] R. J. Walters et al., Proceedings of $37^{\text {th }}$ IEEE PVSC (2011) p. 000122.

[2] M. P. Lumb et al., Proceedings of SPIE (2012) p. 8256.

[3] L. C. Hirst, et al., J. Appl. Phys 117 (2015) p. 215704.

[4] M. González, et al., Proceedings of $42^{\text {nd }}$ IEEE PVSC (2015).

[5] S. Tomasulo, et al., Proceedings of $42^{\text {nd }}$ IEEE PVSC (2015).

[6] N. Baladés, et al., Applied Surface Science 395 (2017) p. 98.

[7] J. Hernández-Saz, et al., Nanotechnology 27 (2016) p. 305402.

[8] This research was performed while the author held an NRC Research Associateship award at the

U.S. Naval Research laboratory.
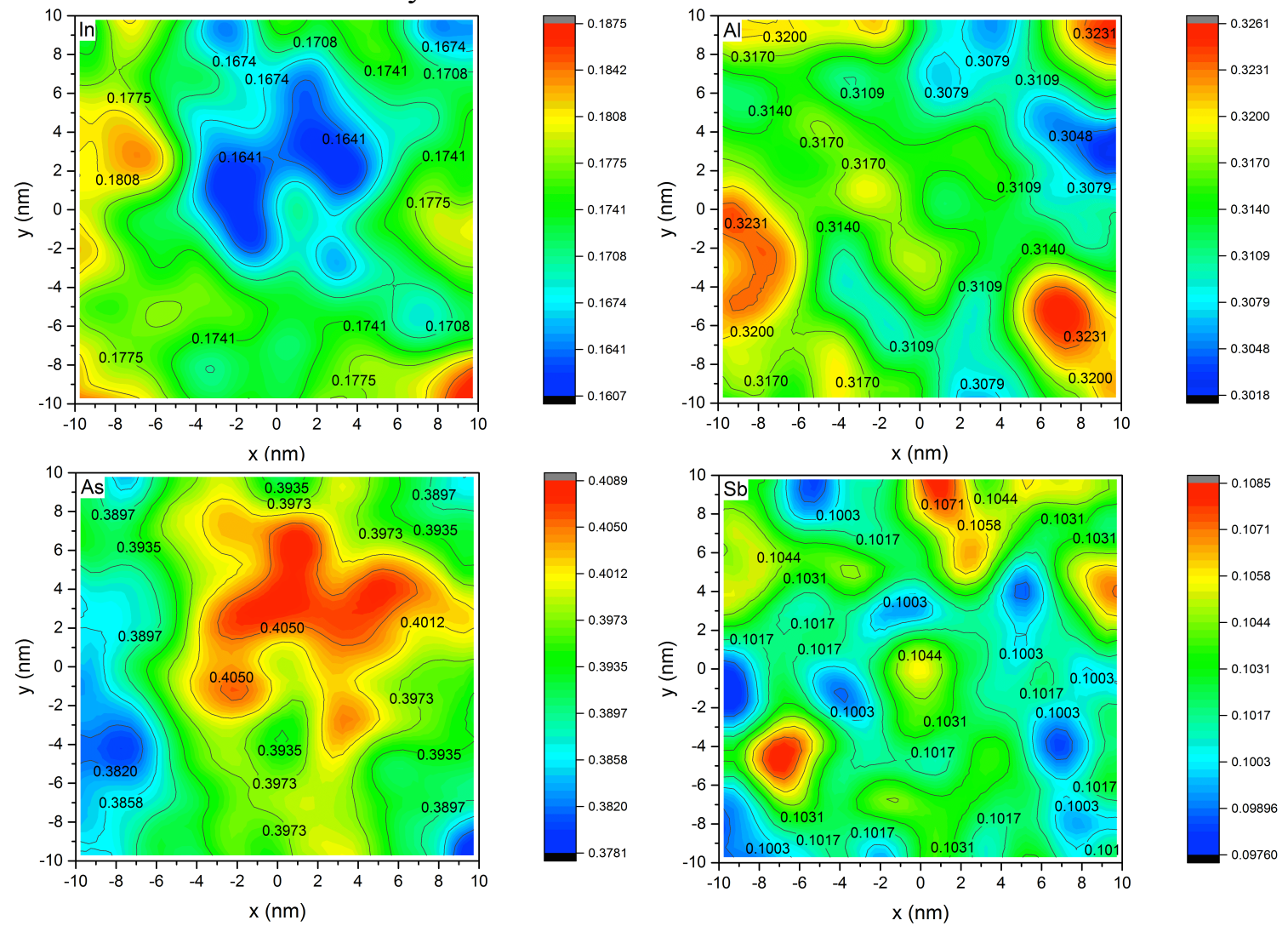

Figure 1. 2D concentration contour maps of $\mathrm{In}, \mathrm{Al}, \mathrm{As}$, and $\mathrm{Sb}$, respectively, looking down the growth direction, for a region of interest $20 \times 20 \times 65 \mathrm{~nm}^{3}$. In and As appear to display anti-clustering behavior. 